\title{
Imidazolium-type anion exchange membranes for improved organic acid transport and permselectivity in electrodialysis
}

Matthew Jordan

Argonne National Laboratory

Tanmay Kulkarni

Penn State University

Dodangodage Senadheera

Louisiana State University https://orcid.org/0000-0003-2393-5952

Revati Kumar

Louisiana State University

Yupo Lin

Argonne National Laboratory

Christopher Arges ( $\sim$ cga5126@psu.edu )

Penn State University

Article

Keywords:

Posted Date: January 11th, 2022

DOI: https://doi.org/10.21203/rs.3.rs-1244464/v1

License: () (1) This work is licensed under a Creative Commons Attribution 4.0 International License.

Read Full License

Version of Record: A version of this preprint was published at Journal of The Electrochemical Society on April 5th, 2022. See the published version at https://doi.org/10.1149/1945-7111/ac6448. 


\title{
Imidazolium-type anion exchange membranes for improved organic acid transport and permselectivity in electrodialysis
}

\author{
Matthew L. Jordan ${ }^{a, c}$, Tanmay Kulkarni ${ }^{d}$, Dodangodage Ishara Senadheera ${ }^{b}$, Revati Kumar ${ }^{b}$, \\ Yupo J. Lin ${ }^{*}$, and Christopher G. Arges ${ }^{d^{*}}$ \\ ${ }^{a}$ Cain Department of Chemical Engineering, Louisiana State University, Baton Rouge, LA 70803 \\ ${ }^{b}$ Department of Chemistry, Louisiana State University, Baton Rouge, LA 70803 \\ ${ }^{c}$ Applied Materials Division, Argonne National Laboratory, Lemont, IL 60439 \\ ${ }^{d}$ Department of Chemical Engineering, Pennsylvania State University, University Park, PA \\ 16802 \\ *corresponding authors: chris.arges@psu.edu yplin@anl.gov
}

\begin{abstract}
Most commercial anion exchange membranes (AEMs) deploy quaternary ammonium moieties. Alternative cation moieties have been explored in AEMs for fuel cells, but there are no studies focused examining alternative tethered cations in AEMs for ionic separations - such as organic acid anion transport via electrodialysis. H-cell and conductivity experiments demonstrate that tethered benzyl 1-methyl imidazolium groups in polysulfone AEMs enhance lactate conductivity by $49 \%$ and improved lactate anion flux by $24 \mathrm{x}$ when compared to a quaternary benzyl ammonium polysulfone AEM. An electrodialysis demonstration with the imidazoliumtype AEM showed a 2x improvement in lactate anion flux and 20\% improvement in permselectivity when benchmarked against the quaternary ammonium AEM. Molecular dynamics and 2D NOESY NMR revealed closer binding of lactate anions to the imidazolium cations when compared to the quaternary ammonium cation. It is posited that this closer binding is responsible to greater flux values observed with imidazolium-type AEM.
\end{abstract}




\section{INTRODUCTION}

Organic acids are used in a range of industrial applications such as the food and beverage industry, biopolymers and biofuels, as well as pharmaceuticals ${ }^{1-4}$. There has been growing interest to produce organic acids through biological pathways for use as a renewable feedstock rather than through the traditional chemical synthesis pathways from fossil resources (e.g., petroleum and natural gas). For example, growth in lactic acid production has been particularly driven by polylactic acid (PLA) plastics which is a biodegradable biopolymer that can be made from a renewable feed stock ${ }^{5,6}$. PLA plastics can display mechanical properties like polyolefinbased plastics for several, but not all, applications. The ester bond in PLA is labile and can be hydrolyzed down to the basic monomers, which can be then recovered and repolymerized to make PLA. Hence, bio-based plastics from organic acids is important to circular economy initiatives that aim to curtail the deleterious effects of non-recycled plastics generated from fossil resources.

Various separation processes have been used to separate and purify organic acids. Precipitation is commonly used due to its simplicity but the process produces hazardous wastewater ${ }^{5}$ that is cumbersome to manage. Precipitation occurs through the addition of a base, that neutralizes the acid leading to an organic anion-cation salt that is insoluble and can be recovered. Notably, the production of one ton of lactic acid with precipitation produces one ton of sodium sulfate byproduct. This approach poses serious environmental waste implications ${ }^{7}$ Solvent extraction has also been widely used for a range of organic acids such as citric, lactic and succinic acids, but the final product purity is limited and this approach employs hazardous solvents ${ }^{4}$. Adsorption has also been investigated but the sorbent lifetime is often short ${ }^{8,9}$ 
necessitating regeneration and leads to excessive solvent waste. Distillation is another method that can yield pure products, but it is the most energy intensive separation technique to employ ${ }^{10}$.

Petroleum-derived organic acids are produced in $80-90 \%$ titers while fermentation broths are limited to $10-20 \%$ titers due to product inhibition and $\mathrm{pH}$ sensitivity of the host organisms. Concentration and dewatering strategies are therefore necessary for capturing the organic acids from fermentation broths to compete in the market against a petroleum-derived alternative. The full range of membrane separations has been applied for organic acid concentration such as reverse osmosis, electrodialysis (ED) and ultrafiltration ${ }^{11-14}$. However RO and ultrafiltration are economically impractical due to the relatively dilute concentrations of bioderived organic acids ${ }^{15}$ and the amount of water that has to be removed from the broth. Organic acids are valuable weakly-ionizable species that can be selectively removed by anion exchange membranes (AEMs) from the fermentation broth media - which is primarily composed on non-ionized constituents. Electrochemical separation platforms, such as electrodialysis, have therefore stood out as a notable separation process for selectively purifying and concentrating organic acids, such as lactic acid. The major advantages of an electrochemical separation technique for organic acid capture are the efficient utilization of the applied current for targeting only the desired acid species and achieving a high product purity that mitigate additional downstream separations. Additionally, electrochemical separation processes are often operated at room temperature and can be powered on renewable energy sources like solar and wind.

To date, the major limitations for ion-exchange membrane used for organic acid separations have been limited flux rates and selectivity for the organic acid/organic acid anions over competing inorganic salts and membrane cost. From a historical perspective, commercial electrodialysis membranes have been primarily developed for desalination and there is a lack of 
knowledge for the appropriate design of ion-exchange membranes for organic acid separations.

The earliest work on characterizing acid transport properties through AEMs primarily considered commercial membranes ${ }^{16-24}$. More recent work has explored thin film coatings ${ }^{25,26}$, nanostructured side chains ${ }^{27}$, and compositive membranes ${ }^{28}$ to improve acid permeation and selective properties of AEMs. Notably, these previous studies have all featured traditional quaternary ammonium and pyridinium moieties in the AEMs.

Most commercial AEMs utilize tethered quaternary ammonium groups for anion exchange and anion conduction. The only commercial AEM that deploys a non-quaternary ammonium group is the Sustainion® AEM sold by Dioxide Materials that feature imidazolium cations. These materials have been designed for electrochemical reduction of carbon dioxide $\left(\mathrm{CO}_{2}\right)$ to value-added chemicals ${ }^{29-31}$. Alternative cation head groups for AEMs have been explored primarily by academic and national labs for fuel cell applications ${ }^{32,33}$, but these studies have not focused on exploring the advantages of alternative head groups for selective ionic separation applications. Manipulating the molecular interactions between the cation head group and target species are one tool that can be used for designing selective membranes for electrochemical separations. Another notable approach in electrochemical separations are redox active polymers using capacitive deionization platforms ${ }^{34-36}$, but these materials have not been investigated for organic acid capture and purification.

A major limitation for widening the applications of electrodialysis for organic acid separations is the inability of the technology to discriminate against ions of similar charge and valence. Hence, desalination of synthetic saline solutions via electrodialysis is a straightforward activity. Targeting one type of ion from a mixture of ions with the same and different valences poses significant separation challenges with conventional electrodialysis (Figure 1a). The 
process conveyed in Figure 1a depicts removal and concentration of organic acid anions along with competing inorganic salt ions such as phosphate and sulfate. Representative liquid mixtures in bioprocesses often contain both organic acids and phosphate and sulfate anions and these inorganic anions can poison the catalyst used in downstream unit operations for the production of renewable fuels, chemicals, and materials ${ }^{37}$. Hence, it is imperative to employ separation processes that selectively remove organic acid anions over inorganic anions. An ideal AEM for acid extraction from fermentation broths (Figure 1b) provides high permeability of the acid anion while mitigating crossover of competing inorganic anions.

In this work, lactic acid anion transport and permselectivity were investigated in Udel ${ }^{\circledR}$ poly(arylene ether sulfone) (PSf) AEMs with imidazolium and quaternary ammonium groups (Figure 1c and 1d, respectively). The polymer backbone chemistry as well as the ion-exchange capacity were controlled precisely to ensure that any changes in transport properties were solely attributed to the cation head group chemistry. The impetus for examining imidazolium groups was inspired by $\mathrm{CO}_{2}$ electrolysis and separations literature showing that imidazolium groups in anion exchange ionomer electrode binders and poly(ionic liquid) membranes promote $\mathrm{CO}_{2}$ reduction kinetics and $\mathrm{CO}_{2}$ uptake ${ }^{29,38-40}$. The $\mathrm{CO}_{2}$ molecule has some chemical similarity to the carboxylate moiety in organic acid anions. Ex-situ membrane transport experiments showed a 24x increase in lactate anion permeation in quaternary benzyl imidazolium PSf (QIPSf) AEMs over quaternary benzyl ammonium PSf (QAPSf) AEMs. Ionic conductivity of QIPSf AEMs with lactate anions were found to be $49 \%$ higher than QAPSf AEMs. Electrodialysis experiments with a single diluate chamber also substantiated greater removal rates and permselectivity of the lactate anions in the presence of sulfate and phosphate competing anions with QIPSf AEMs over QAPSf AEMs. 
To better understand the experimental observations, classical molecular dynamics (MD) simulations and density functional theory (DFT) simulations, as well as 2D NMR Nuclear Overhauser Effect Spectroscopy (NOESY), were deployed to probe the interactions of lactate with the different cation group chemistries. It was uncovered that the lactate anion was located closer to the imidazolium moiety when compared to quaternary ammonium moiety and that the lactate ion interacted differently with the cation group depending on its chemical structure. The simulations and NMR spectroscopy motivate future research activities probing how the proximity of the counterion and its interaction with the tethered cation alter transport rates in AEMs. The working hypothesis posits that lactate anions bind closer to the imidazolium polymer backbone, but when solvated, accelerate the hopping mechanism from one tethered cation to another leading to greater transport rates in the AEM. 


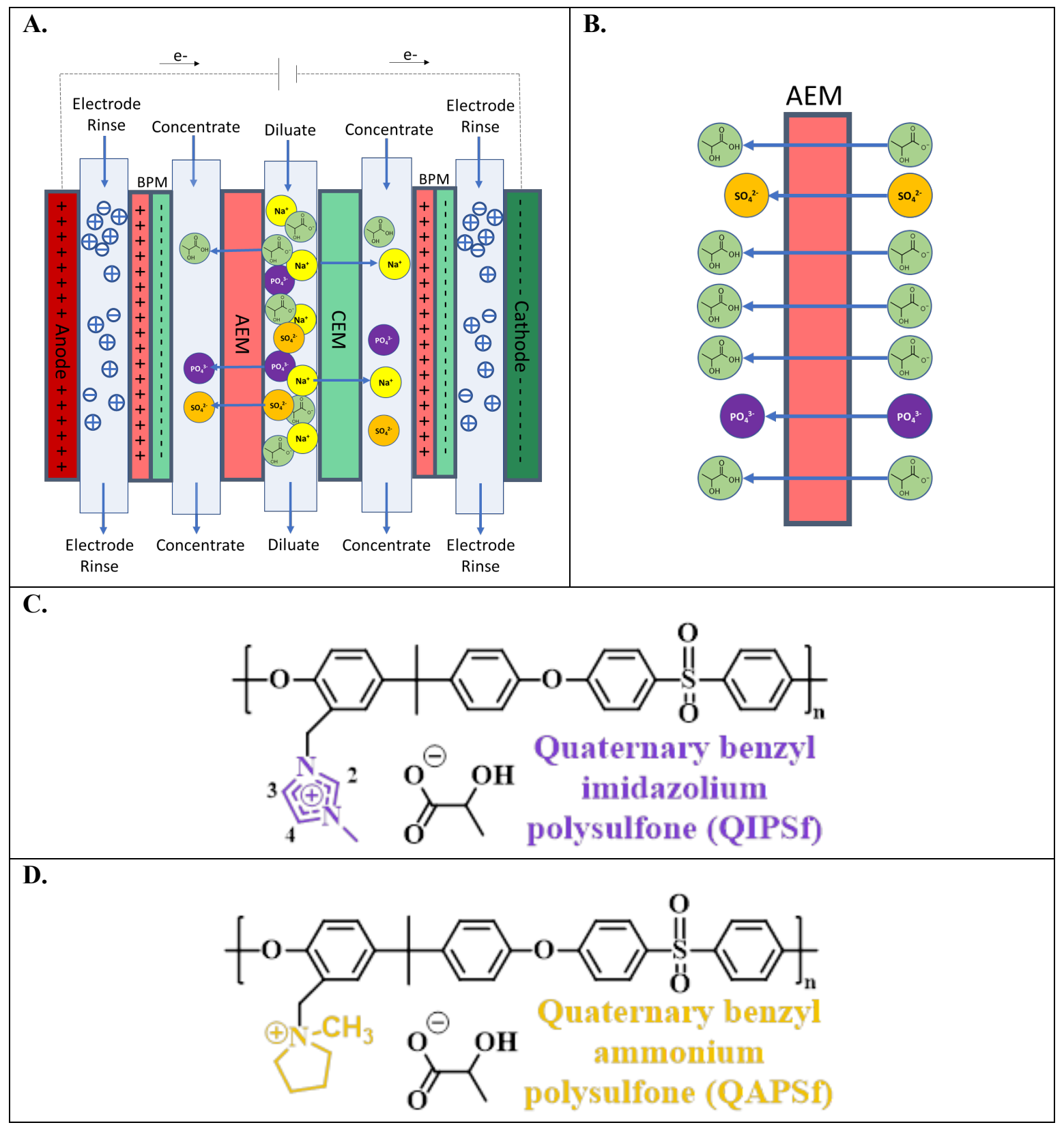

Figure 1. (a) Concentration of lactate ions from single cell pair electrodialysis device. The illustrated cell conveys the current state of electrodialysis that has difficultly in discriminating between lactate and inorganic salts (sulfate or phosphate). (b) Ideal AEM that selectively removes greater lactate anion over competing ions like phosphate and sulfate. The chemical structure of AEMs investigated in this work: (c) quaternary benzyl imidazolium Udel ${ }^{\circledR}$ poly(arylene ether sulfone) (QIPSf) and (d) quaternary benzyl ammonium $\mathrm{Udel}^{\circledR}$ poly(arylene ether sulfone) (QAPSf). 


\section{RESULTS AND DISCUSSION}

AEM permeability, conductivity, and acid uptake experiments

The permeability coefficient $\left(\mathrm{P}_{\mathrm{a}}\right)$ for lactic acid were characterized with a concentration cell experiment (see Figure S1). The lactate crossover rates into the permeate chambers are plotted in Figures 2a. The steady state acid permeability $\left(\mathrm{P}_{\mathrm{a}}\right)$ for lactate through the AEMs was calculated using Equation 1 listed in the Methods section. A 24x increase in the permeate concentration compartment occurred with the QIPSf AEM when compared to the QAPSf AEM.

Although concentration gradient driven permeability experiments are useful for comparing transport rates of an ion through an ion-exchange membrane, it is important to note that electrodialysis is an electric field driven separation and thus assessing AEM conductivity is also important for assessing an AEM's potential for an effective separation in electrodialysis. Figure 2b reports the ionic conductivity of the QIPSf and QAPSf AEMs with a lactic acid supporting electrolyte (Figure 2b). The QIPSf had an 49\% ionic conductivity in lactic acid supporting electrolytes when compared to QAPSf AEMs. The permeability and conductivity are listed in Table 1.

Permeability coefficients are a product of equilibrium partitioning coefficient multiplied by the diffusion coefficient $\left(P_{\mathrm{a}}=K_{\mathrm{a}} D_{\mathrm{a}}\right)$. To better understand permeability and conductivity observations of QIPSf versus QAPSf, equilibrium partitioning experiments were performed to discern if greater ion-partitioning (i.e., greater acid uptake) was responsible for the improved lactic acid/lactate anion transport with QIPSf. Figure 2c reports the equilibrium concentrations of the lactic acid in the external solution and in the AEM samples. These experiments were performed by first preparing three different concentrations of the lactic acid and bringing these solutions into contact with the AEM sample. After an extended period (i.e., 48 hours), the 
concentration of organic acid remaining in the external solution and the acid present in the AEM was measured via gravimetric and quantitative NMR (qNMR) approaches. The slope of the lines presented in the graphs were used to calculate, $K$, which is the equilibrium partitioning coefficients for the various organic acids with AEM samples. The values for $K$ are listed in Table 1. $K$ was calculated by both gravimetric and qNMR and it was statistically the same for QAPSf and QIPSf indicating that the imidazolium groups did not promote greater acid uptake. Given this observation, it was inferred that the increased lactate anion permeability of QIPSf over QAPSf arises from greater diffusion coefficient of the lactate anion in QIPSf versus QAPSf. Additionally, the greater diffusivity of the lactate anion in QIPSf accounts for the higher ionic conductivity for QIPSf over QAPSf as conductivity is linearly commensurate with the diffusion coefficient (Equation 2).

$$
\kappa=F^{2} \cdot \sum_{i=1}^{n} z_{a}^{2} \cdot C_{a} \cdot \frac{D_{a}}{R T}
$$




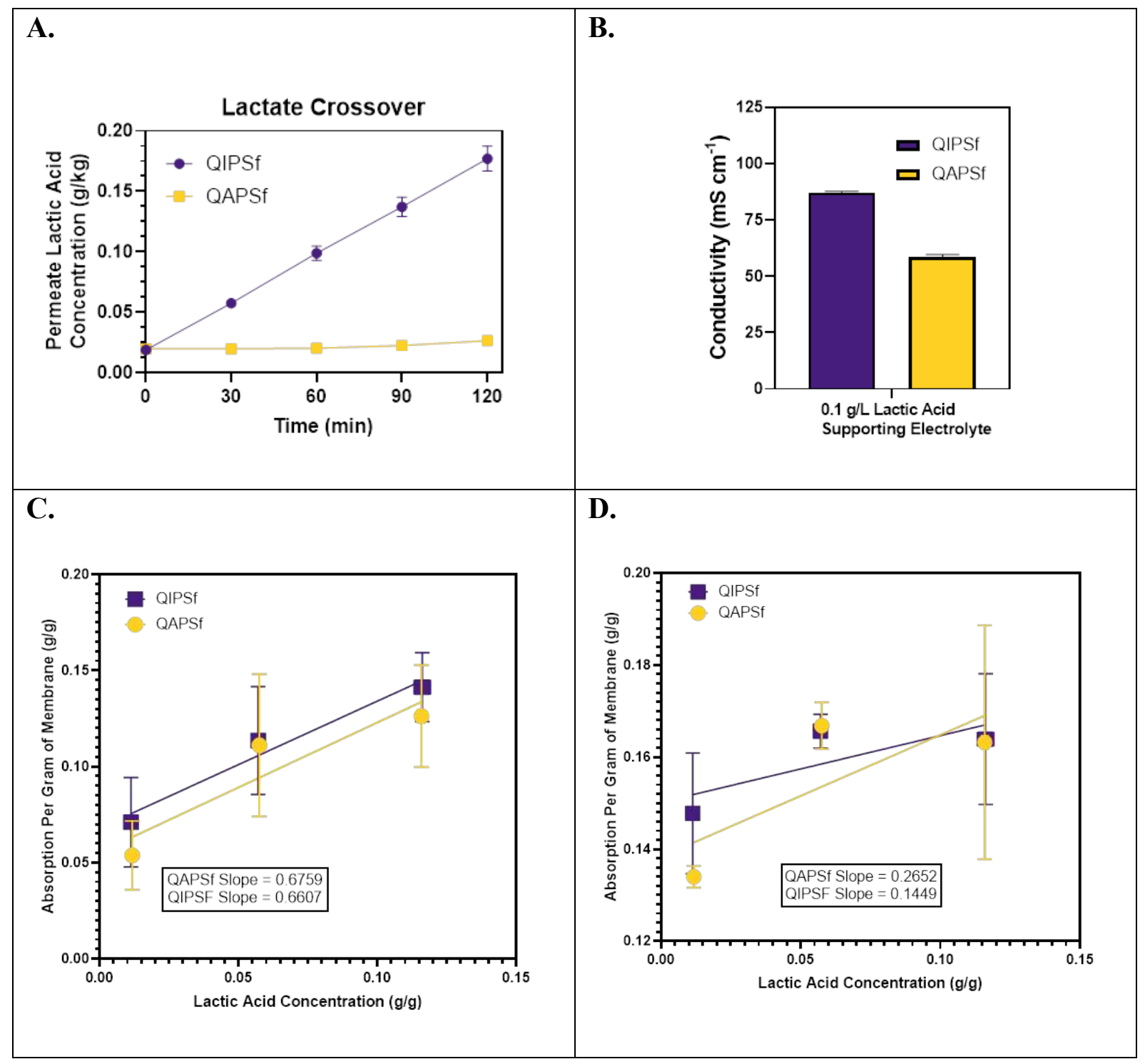

Figure 2. (a) Permeability crossover results for lactate across the AEM with QIPSf and QAPSf. (b) Ionic conductivity values of QIPSf and QAPSf in $0.1 \mathrm{~g} \mathrm{~L}^{-1}$ lactic acid supporting electrolyte. Equilibrium absorption of lactic acid per gram of membrane versus supporting lactic acid solution concentration by (c) a gravimetric measurement and (d) a quantitative NMR measurement. Error bars represent the standard error between triplicate measurements $(n=3)$.

Table 1. Membrane characterization for QIPSf and QAPSf

\begin{tabular}{|c|c|c|c|c|}
\hline $\begin{array}{c}\text { AEM } \\
\text { Type }\end{array}$ & $\begin{array}{c}\text { Conductivity in } \mathbf{~ 0 . 1} \mathbf{~ g ~ k g}^{-1} \\
\text { lactic acid } \\
\left(\mathbf{m S ~ c m}^{-1}\right)\end{array}$ & $\begin{array}{c}\text { Gravimetric lactic } \\
\text { acid equilibrium } \\
\text { coefficient }\end{array}$ & $\begin{array}{c}\text { qNMR lactic acid } \\
\text { equilibrium } \\
\text { coefficient }\end{array}$ & $\begin{array}{c}\text { Lactate } \\
\text { permeability } \\
\left.\text { (cm }^{\mathbf{2}} \mathbf{~ s}^{-1}\right)\end{array}$ \\
\hline QIPSf & $87.3 \pm 0.4$ & $0.7 \pm 0.2$ & $0.14 \pm 0.07$ & $(1.3 \pm 0.1) \times 10^{-7}$ \\
\hline QAPSf & $58.5 \pm 1.1$ & $0.7 \pm 0.2$ & $0.3 \pm 0.1$ & $(5.0 \pm 0.3) \times 10^{-9}$ \\
\hline
\end{tabular}




\section{Electrodialysis with different AEMs using synthetic fermentation broths}

The rate of acid capture and permselectivity of the two different PSf AEMs were compared with a one cell-pair electrodialysis unit that is illustrated in Figure 1a. A synthetic fermentation broth containing $33 \mathrm{~g} \mathrm{~kg}^{-1}$ lactic acid, $1.25 \mathrm{~g} \mathrm{~kg}^{-1}$ sodium sulfate and $0.72 \mathrm{~g} \mathrm{~kg}^{-1}$ sodium phosphate was used as the feed solution for the diluate chamber and a $10 \mathrm{~g} \mathrm{~kg}^{-1}$ sodium chloride solution in the concentrate chamber. The concentrations of the lactate, phosphate, and sulfate components in the diluate and concentrate compartments as a function of time during the ED experiment are given in Figures S2a, S2b, and S2c in the SI. The change in the concentration of the said anion components over time were used to compute flux rates, permselectivity and energy consumption values, which are given in Table 2, using Equations 7, $\mathbf{9}$ and $\mathbf{1 0}$ (listed in the Methods section).

Table 2. Transport properties for anion exchange membranes

\begin{tabular}{|c|c|c|c|c|c|c|}
\hline $\begin{array}{l}\text { AEM } \\
\text { Type }\end{array}$ & $\begin{array}{l}\mathbf{J}_{\text {lactic acid }} \\
\left(\mu \mathrm{mol} \mathbf{c m}^{-2}\right. \\
\left.\text { min }^{-1}\right)\end{array}$ & $\begin{array}{l}\text { J } \begin{array}{l}\text { sulfate } \\
\left(\mu \mathrm{mol} \mathrm{cm}^{-2}\right. \\
\left.\mathrm{min}^{-1}\right)\end{array} \\
\end{array}$ & $\begin{array}{l}\text { J phosphate } \\
\left(\mu \mathrm{mol} \mathbf{c m}^{-2}\right. \\
\left.\min ^{-1}\right)\end{array}$ & Plactic acid $\mathrm{SO}^{2-}$ & Plactic Acid PO4 $^{3-}$ & $\begin{array}{l}\text { Energy consumption } \\
\text { per kg lactic acid } \\
\left(\mathrm{kWh} \mathrm{kg}^{-1}\right)\end{array}$ \\
\hline QIPSf & 5.91 & 0.061 & 0.31 & 0.39 & 1.36 & 0.704 \\
\hline QAPSf & 2.97 & 0.038 & 0.20 & 0.31 & 1.10 & 1.83 \\
\hline$\%$ change & $+99 \%$ & $+62 \%$ & $+54 \%$ & $+27 \%$ & $+23 \%$ & $-62 \%$ \\
\hline
\end{tabular}

The single-cell pair ED experiments with a QIPSf showed a 99\% higher lactate flux when compared to using QAPSf AEM (Figure 3a). Using a QIPSf AEM also promoted higher sulfate and phosphate fluxes, 62\%, and 54\%, respectively, when compared to the QAPSf AEM. Despite this unwanted side effect, the permselectivity of lactate over phosphate and sulfate for the QIPSf versus the QAPSf was greater because the increase in phosphate and sulfate anion fluxes in QIPSf was not commensurate with increase in lactate anion flux (Figure 3b). Furthermore, the 
increased flux of lactate reduced the energy consumption for lactate capture by $62 \%(1.83 \mathrm{kWh}$ $\mathrm{kg}^{-1}$ versus $0.704 \mathrm{kWh} \mathrm{kg}^{-1}$ for QAPSf and QIPSf, respectively, Figure 3c). Increasing the organic acid anion flux across AEMs is the main contributor to reducing the overall energy consumption for primary acid extraction technologies from fermentation broths as the electrodialysis cell is operated at constant voltage.

Future work will look to crosslink the QIPSf AEM and to make it thicker to enhance the permselectivity for lactate over other ions in fermentation broths. This will reduce the lactate anion flux. However, this approach will have a greater impact on reducing competing anion transport and crossover. This is important because sulfate disrupts downstream processes via catalyst poisoning. Hence, complete elimination of sulfate transport in the concentrate chamber is a high priority in organic acid capture from fermentation broths. The tradeoff between permeability and selectivity is well-known in the general area of membrane separations (e.g., Robeson plots ${ }^{41}$ ). 


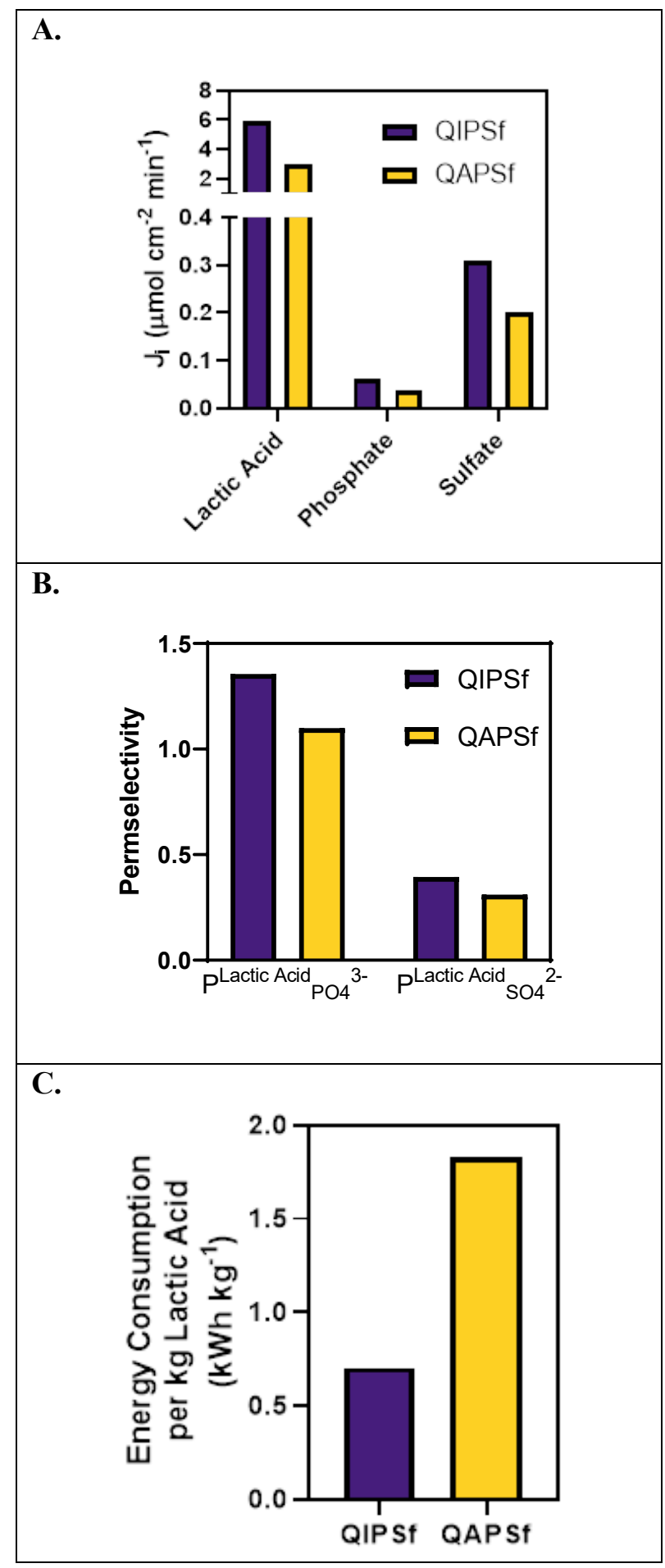

Figure 3. Results from single-cell ED experiments: (a) Ionic flux of lactic acid, phosphate and sulfate across QIPSf and QAPSf AEMs. (b) Permselectivity of lactic acid capture rate over phosphate and sulfate capture. (c) Energy consumption for lactic acid capture. 
Understanding the observed differences in organic acid anion transport in AEMs using molecular simulations and NOESY NMR spectroscopy

The previously presented research results unequivocally support greater lactate anion in transport in QIPSf over QAPSf AEMs. The only difference in these membranes are the tethered cation chemistries: quaternary benzyl imidazolium and quaternary benzyl n-methyl pyrrolidinium (a type of quaternary ammonium). The AEM backbone chemistries, tethering strategy, and IEC are the same.

To determine how the imidazolium group may favorably promote lactate anion transport, MD simulations were performed to look at the spatial distribution (i.e., radial distribution function) of the lactate counterion in relation to the different tethered cationic groups. The MD simulations consisted of PSf backbone chains with tethered imidazolium and quaternary ammonium groups, lactate counterions, and water. These simulations were augmented by quantum DFT calculations of the cation chemistries with the lactate counterion only (i.e., the simulated system in DFT did not contain the polymer backbone or water). Quantum electronic structure calculations provide an accurate description of the interactions between atoms despite the limited accessible time scale and size of the system compared to MD simulations.

The radial distribution function $(\mathrm{g}(\mathrm{r}))$ from MD simulations for the oxygen atoms in lactate's carboxylate group with the carbon and nitrogen atoms in the tethered cation are given in Figure 4a. The imidazolium carbon (-CH-) between the two nitrogen atoms in the imidazolium ring is commonly labeled as $\mathrm{C} 2$ in the literature and its relation to the oxygen atoms in lactate is shown in Figure 4b. The g(r) displays an intense and sharp peak around $3.0 \AA$ for the distribution of oxygens in the lactate around the imidazolium C2 carbon (purple traces labeled $\mathrm{C} 2$ in Figure 4a) indicating a stronger and more structured association when compared to the 
distribution of lactate oxygen atoms around the nitrogen atoms in the imidazolium (purple traces labeled N1 and N2 in Figure 4b). The first peak for the radial distribution functions of the quaternary nitrogen in the ammonium group (labeled N3 in Figure $4 \mathbf{b}$ ) to lactate oxygens appears at $4.5 \AA$ (yellow trace in Figure 4a) and demonstrates that the lactate counterion is further away from the tethered quaternary ammonium when compared to the tethered imidazolium. Additionally, the lactate coordination with the quaternary ammonium nitrogen is less structured because the $\mathrm{g}(\mathrm{r})$ is broader when compared to the $\mathrm{C} 2$ - lactate $\mathrm{g}(\mathrm{r})$ in QIPSf.

The shorter distance between the $\mathrm{C} 2$ in imidazolium to lactate oxygen peak (i.e., $\mathrm{C} 2$ - O (lactate)) compared to the quaternary ammonium nitrogen to lactate (N3 - $\mathrm{O}($ lactate) $)$ is attributed to the planar structure of the imidazolium and its less hindered environment around the ring that allows lactate ions to interact easier. In the case of quaternary ammonium, the lactate ion interaction with the nitrogen is hindered due to the tetrahedral geometry of nitrogen and the methyl/-alkyl groups attached to it. Furthermore, the radial distribution functions of the carbons $\left(-\mathrm{CH}_{2}-\right.$ and $\left.-\mathrm{CH}_{3}\right)$ in the quaternary ammonium to the lactate oxygens (labeled $\mathrm{C} 3$ and $\mathrm{C} 4$ in Figure S3) are less structured with a peak at slightly larger distances compared to the distribution of lactate oxygens around the $\mathrm{C} 2$ in imidazolium. This substantiates that the imidazolium $\mathrm{C} 2$ association to lactate is still the most prominent. 
A.

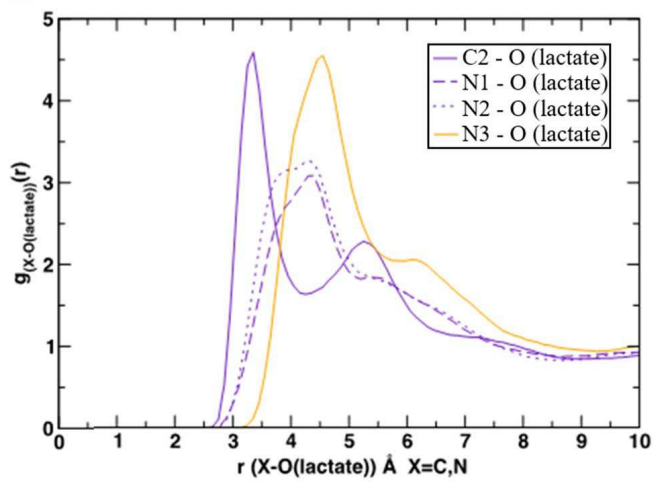

B.

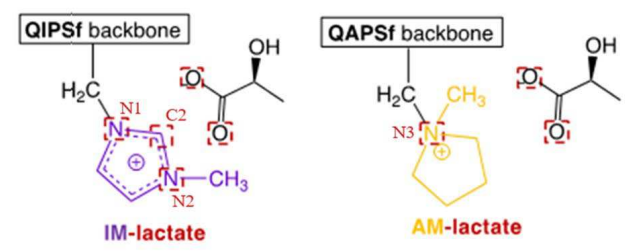

C.

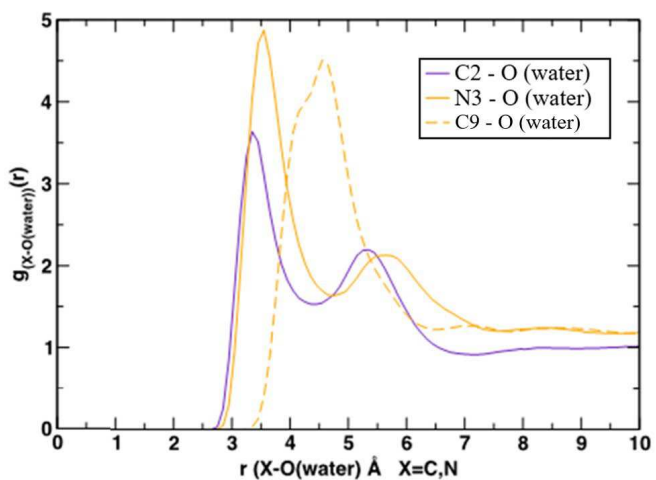

D.

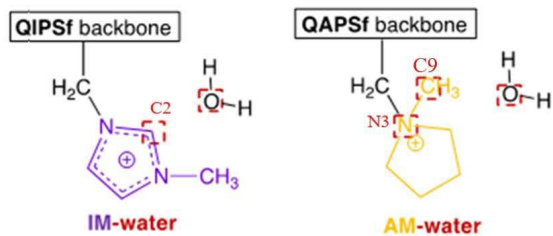

Figure 4. (a) The radial distribution function $g(r)$ between the lactate anion oxygens to the corresponding atoms in the imidazolium (IM) and ammonium cation (AM) group. (b) Illustration of the corresponding atoms (marked by a red square) in the imidazolium ring (IM) and in the quaternary ammonium ring (AM) that were considered for the $\mathrm{g}(\mathrm{r})$ calculation in each system. (c) The radial distribution function $\mathrm{g}(\mathrm{r})$ between the positively charged imidazolium/ammonium groups to the $\mathrm{O}$ atom of water molecules. (d) Illustration of the corresponding atoms (marked by a red square) considered for imidazolium-water and ammonium-water radial distribution functions.

The snapshots taken from each simulation (Figures S4 and S5) reveals that the lactate ions are always positioned above the imidazolium ring such that the oxygens of lactate ions favor the interaction of the $\mathrm{C} 2$ carbon in the imidazolium ring (Figure S4). The snapshots taken from the quaternary ammonium system (i.e., QAPSf) (Figure S5) reveal that the lactate ions are positioned in all possible directions without any preferred direction to the ammonium ring. This spatial arrangement of lactate ions around the ammonium ring may signal weaker interactions between the quaternary ammonium cation and lactate anion.

The hydration levels around the positively charged imidazolium and ammonium groups paired with negatively charged lactate anions were also determined from classical MD 
simulations. The $\mathrm{g}(\mathrm{r})$ calculated between the corresponding atoms in the imidazolium and ammonium cations to the oxygen in water (Figure 4c) reveal that imidazolium cation is solvated with fewer water molecules as compared to the ammonium cation. The first minimum of each radial distribution function in Figure $\mathbf{4 c}$ defines the first hydration shell around the atoms. By considering this cut-off $\left(\mathrm{r}=4.45 \AA\right.$ for the $\mathrm{C} 2$ carbon in imidazolium and $\mathrm{r}=4.75 \AA$ for the $-\mathrm{CH}_{3}$ carbon of quaternary ammonium), the average number of water molecules around the $\mathrm{C} 2$ carbon is $2.10 \pm 0.05$ and around the $\left(-\mathrm{CH}_{3}\right)$ carbon of quaternary ammonium is $2.59 \pm 0.08$. These values signal that there are more waters around the quaternary ammonium cation compared to the imidazolium cation. This observation is also supported from the snapshots taken from imidazolium system (Figure S4) where a smaller number of water molecules are present near the $\mathrm{C} 2$ carbon in imidazolium whereas there are more waters present around the ammonium group in the snapshots taken from the ammonium system (Figure S5).

The larger amount of water molecules around the ammonium cation suggest that the water molecules compete with lactate anions to interact with the quaternary ammonium. This competition may lead to weaker ammonium-lactate association. But, in the case of imidazolium, the imidazolium-lactate association is stronger resulting in a slight decrease in coordinating water molecules as compared to the ammonium case.

The results from MD simulations of polymer chains with tethered cations and mobile lactate anions depend on the accuracy of the force-field parameters used. To further confirm the stronger imidazolium-lactate binding as compared to ammonium-lactate idea, electronic structure calculations using DFT on representative clusters were carried out to compare the binding energy of a lactate anion with an imidazolium cation to the binding energy of a lactate anion with a quaternary ammonium cation. Figure 5 presents the optimized structures, and the binding energy 
values for the two calculations. The lactate-imidazolium binding energy is about $-65.89 \mathrm{kcal} / \mathrm{mol}$ and this value is lower than the lactate-quaternary ammonium binding energy of $-50.45 \mathrm{kcal} / \mathrm{mol}$. These calculations further support the preference of lactate ions towards an imidazolium cation over a quaternary ammonium cation. The optimized distances between the oxygen atoms in the carboxyl group of lactate to the $\mathrm{C} 2$ carbon in imidazolium are $2.97 \AA$ and $1.94 \AA$, respectively, which is consistent with hydrogen bonding interactions - namely the more acidic proton at the C2 carbon position. These interactions are posited to be responsible for the lactate ions being closer to the imidazolium ring. In comparison, the distance between the oxygen atoms in lactate's carboxyl group to the quaternary nitrogen and adjacent methyl group carbon in quaternary ammonium is $3.55 \AA$ and $3.15 \AA$, respectively, which are all greater than $3.0 \AA$. The weaker van der Waals interactions between the quaternary ammonium and lactate are responsible for the lactate anion not binding as close to the quaternary ammonium cation as the imidazolium cation.

(A)

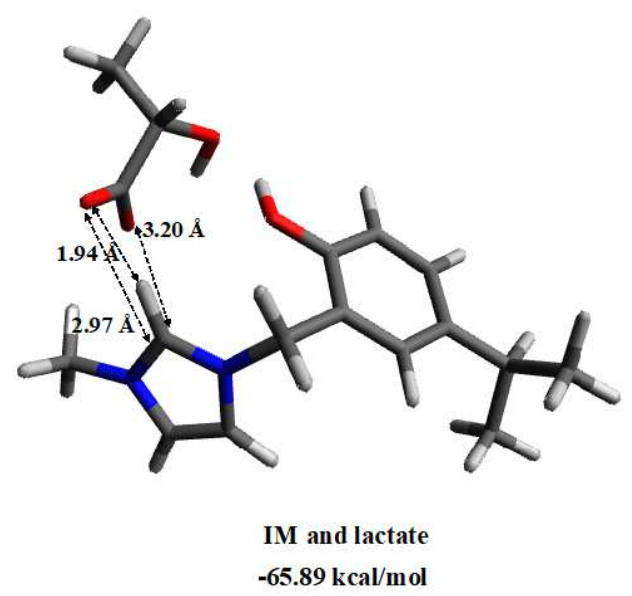

(B)

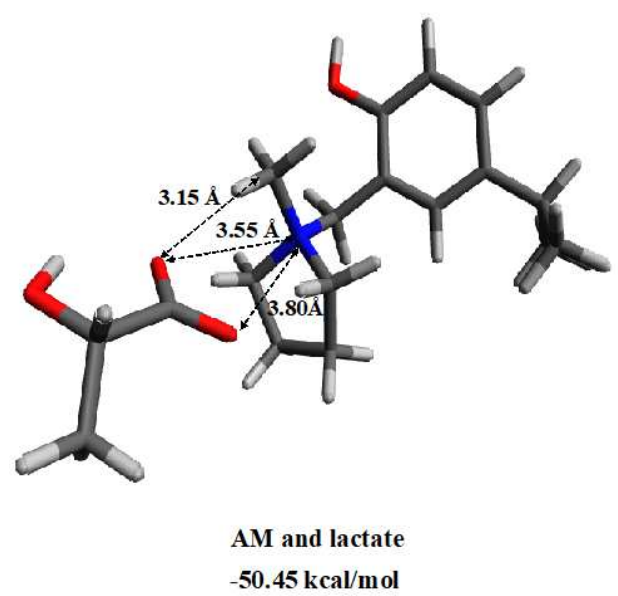

Figure 5. Optimized structures, distances, and binding energies of lactate anions (a) to the imidazolium group and (b) to the quaternary ammonium group calculated using GAUSSIAN at the B3LYP/6-31+g(d) level of theory. Nitrogen, oxygen, hydrogen, and carbon atoms are represented in blue, red, white, and grey colors respectively. 
2D NOESY NMR spectroscopy was performed to complement the molecular simulations and to further test if the lactate anion was bound more closely to the benzyl imidazolium in QIPSf when compared to the quaternary benzyl ammonium in QAPSf. 2D NOESY identifies inter- and intra- through space interactions between proton moieties ${ }^{42}$ and can be used to determine if the counter anion is within $5.0 \AA$ of the tethered cation. Off-diagonal peaks in the 2D NOESY NMR spectra correspond to protons that have through-space interactions and was used to determine which moieties in the tethered cations and lactate anion were near each other.

Figures $6 \mathbf{a}$ and $\mathbf{6 b}$ presents the NOESY spectra of QAPSf and QIPSf containing chloride as the counterion. These control experiments were performed to identify off-diagonal signals (i.e., proton-proton through space interactions) related to QIPSf and QAPSf only. Similar to 2D COSY NMR spectra by Arges et al. for these types of AEMs ${ }^{43,44}$, the off-diagonal peaks seen in the 2D NOESY spectra for QIPSf and QAPSf in the chloride form arise from the protons in the aryl rings of the PSf backbone and appear in the chemical shift region of 7.0 to $8.0 \mathrm{ppm}$.

Figures 6c and 6d provide the 2D NOESY spectra for QIPSf and QAPSf ion-exchanged to the lactate counterion form. The 2D NOESY for lactate is given in Figure S6. No off-diagonal peaks are observed for lactate. Figure 6d identified an off-diagonal peak (labelled as a-m) corresponding to the protons in the methyl moiety of lactate and the $\mathrm{C} 2$ carbon in the imidazolium ring for QIPSf. Figure 6c shows an off-diagonal peak between the tertiary carbon proton (i.e., '-CH-') in the lactate and the methyl group ('- $\mathrm{CH}_{3}$ ') adjacent to the nitrogen in the cyclic quaternary ammonium ring. The off-diagonal peaks observed in the Figures $\mathbf{6 c}$ and $\mathbf{6 d}$ signal that the lactate was within $5.0 \AA$ of the tethered cations in both QIPSf and QAPSf. This conveyed that lactate is bound close to both cations. Interestingly, the through-space interactions for the two systems were different. 
The MD simulation results presented previously showed the $\mathrm{g}(\mathrm{r})$ for carbon atoms with nitrogen and oxygen atoms for QIPSf and QAPSf. The 2D NOESY detected close binding of: i.) protons from the $\mathrm{C} 2$ carbon of imidazolium with the methyl in lactate and ii.) the methyl in the quaternary ammonium with the - $\mathrm{CH}$ - in lactate. The observations from 2D NOESY motivated further analysis from the MD simulations; namely, the $\mathrm{g}(\mathrm{r})$ for the carbon atoms containing the protons that displayed through-space interactions from NOESY experiments (Figures 6e and 6f).

From the $\mathrm{g}(\mathrm{r})$ for QAPSf, the distribution of the tertiary carbon ('-CH-') in lactate around the methyl group in quaternary ammonium was found to be greatest and with the smallest binding distance (solid orange line labeled $\mathrm{i}-\mathrm{g}$ ). This finding is consistent with the experimental NOESY results where an off-diagonal peak was observed corresponding to the protons associated with lactate's tertiary carbon and the quaternary ammonium's methyl group.

The distribution between the lactate tertiary carbon and lactate methyl group around the C2 carbon in QIPSf was also investigated from the $\mathrm{g}(\mathrm{r})$ simulation data. The $\mathrm{g}(\mathrm{r})$ shows that the first order distribution of the methyl groups in lactate around the $\mathrm{C} 2$ carbon in the imidazolium group occurs at 3.9 to 4 angstroms (labelled $\mathrm{a}-\mathrm{m}$ in Figure 6e) and this interaction occurs closer than the first order peak for the tertiary carbon-imidazolium interaction (labelled $a-j$ ). Although there are more tertiary lactate carbons surrounding the $\mathrm{C} 2$ carbon in imidazolium, the interaction is approaching the 5-angstrom cutoff for NOESY through-space detection (as well as being a single proton interaction) which makes it difficult to detect. Therefore, the population of methyl groups from the lactate around the $\mathrm{C} 2$ carbon in imidazolium is detected by the NOESY experiments due to being sufficiently below the 5 -angstrom threshold. 


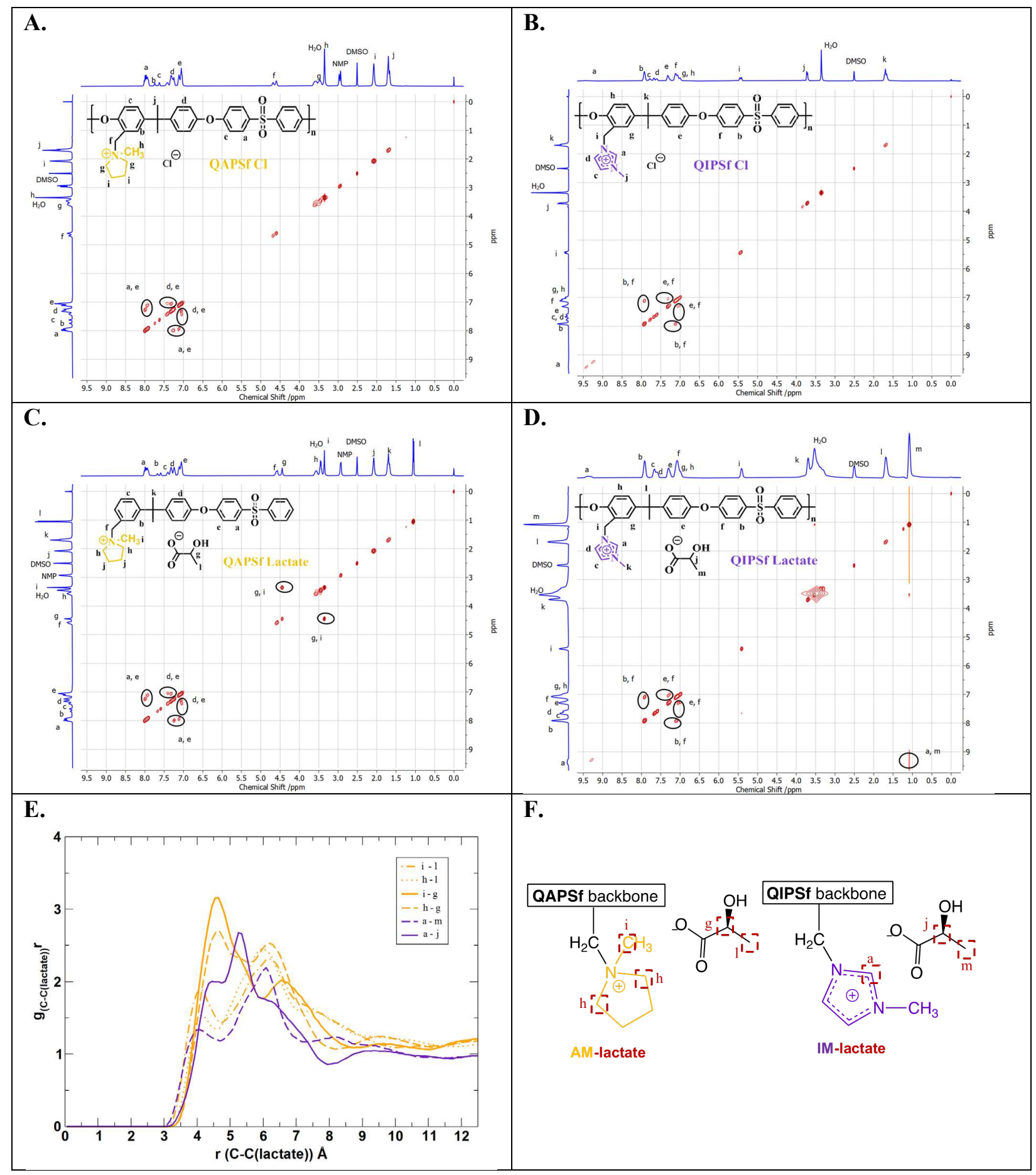

Figure 6. 2D NOESY spectra of (a) QAPSf and (b) QIPSf in chloride counterion and (c) QAPSf and (d) QIPSf in lactate counterion form. (e) The radial distribution function $(\mathrm{g}(\mathrm{r}))$ between the lactate tertiary carbon and methyl group to corresponding locations in the imidazolium (IM) and quaternary ammonium cation (AM) groups where through-space interactions were observed in the 2D NOESY spectrums. (f) Illustration of the corresponding atoms (marked by a red square) in the imidazolium ring (IM) and in the quaternary ammonium (AM) that were considered for the $g(r)$ calculation. 


\section{DISCUSSION}

Permeability and ionic conductivity measurements combined with qNMR and gravimetric ion-partitioning measurements unequivocally demonstrated that quaternary imidazolium groups promote lactate diffusion over quaternary ammonium groups in PSf-based AEMs. The materials property observations translated to improved lactate fluxes and greater permselectivity when utilizing a QIPSf AEM over a QAPSf AEM in a single-cell electrodialysis unit fed with a model fermentation broth. The implications of these results will lead to electrodialysis with a smaller membrane area requirement and a reduced capital cost for the separation platform. The greater flux of organic acid anions through imidazolium-type of AEMs also reduced the energy consumption for the organic acid recovery in electrodialysis.

To uncover the origins of the better organic acid anion diffusion in QIPSf over QAPSf AEMs, classical MD and DFT simulations were performed in addition to 2D NOESY NMR. These simulations revealed that the lactate counteranion was closer to the benzyl imidazolium group when compared to the quaternary benzyl ammonium group. The 2D NOESY NMR further showed that the lactate anion was within $5.0 \AA$ of both tethered cation types. However, the moieties in the lactate that interact with the tethered cations were different: the methyl group in lactate was closer to the $\mathrm{C} 2$ in the imidazolium while the - $\mathrm{CH}$ - in the tertiary carbon of lactate interacted with the methyl group in the quaternary ammonium cation. The reduced binding distance and binding energy for lactate with imidazolium, in addition to reduced interference by water molecules, when compared to lactate with quaternary benzyl ammonium is responsible for the improved lactate anion transport rate with a QIPSf AEM over a QAPSf AEM.

The smaller distance between lactate and quaternary imidazolium compared to lactate and quaternary ammonium initially suggests a stronger interaction between imidazolium and 
lactate that may impede lactate anion transport under a chemical potential or electrochemical potential gradient driving force. However, it should be noted that condensed counterions in ionexchange membranes have been observed to diffuse 2 to $2.5 \mathrm{x}$ faster than non-condensed counterions in highly-charged and crosslinked ion-exchange membranes ${ }^{45}$. A condensed counterion can be considered a cation in close proximity, such as a distance less than the Bjerrum length ${ }^{46}$, to a tethered ionic group. This is sort of counterintuitive as fully (or better) dissociated counterions would be more conducive for transport in the polymer matrix. However, if there is a sufficient density of tethered charge/ionic groups along the polymer backbone, and the ionic groups are solvated ${ }^{47}$, counterion ion transport along the polymer chain can be effective for promoting transport.

Although 2D NOESY substantiated that both cations were close to the lactate, it was uncovered that the cation groups interact with different moieties in the lactate anion. One potential explanation is that the interaction and tighter binding of '-CH-' in lactate to the methyl in the quaternary ammonium makes it more difficult for lactate to migrate when compared to the lactate methyl being closer to the $\mathrm{C} 2$ carbon in the imidazolium ring. Future work will study the anion transport mechanisms along the backbone and relate them to the binding with different chemical moieties in the cation and counterion. More fundamental research between the said molecular interactions and their role on transport in ion-exchange membranes is needed for improving the performance and energy efficiency of electrochemical separation platforms like electrodialysis. 


\section{METHODS}

Materials

$\mathrm{Udel}^{\circledR}$ poly(arylene ether) sulfone (PSf), paraformaldehyde, chlorotrimethylsilane, tin(IV) chloride, 1-methylpyrrolidine, 1-methylimidazole were sourced from Acros Organics, chloroform and methanol from VWR Chemicals, and were used in the CMPSf synthesis, which is the precursor to make QAPSf and QIPSf. Sodium 3-(Trimethylsilyl)-1-propanesulfonate (DSS) was attained from TCI America. Deuterated dimethyl sulfoxide (d6-DMSO) was acquired from Alfa Aesar and it was used in quantitative NMR measurements for equilibrium acid uptake experiments. $60 \mathrm{wt} \%$ sodium DL-lactate was purchased from TCI America. Deionized water (DI $\mathrm{H}_{2} \mathrm{O}, 18.2 \mathrm{M} \Omega,<20 \mathrm{ppb}$ TOC) was withdrawn from a Milli-Q Millipore Elix 10 system.

Commercially available cation exchange and bipolar membranes were obtained from Ameridia (Neosepta CMX and BP; ASTOM Corporation, Tokyo, Japan) and were used for the ED stack measurements.

Synthesis of QIPSf and QAPSf AEMs

PSf was chloromethylated by a Friedel-Crafts reaction based on the procedure reported by Arges and co-workers ${ }^{48}$. The procedure is given in the SI and is shown in Figure S7.

Tethered cation groups were installed on to CMPSf via nucleophilic substitution to prepare the quaternary benzyl ammonium and imidazolium polysulfone AEMs. The reaction commenced by dissolving CMPSf in n-methylpyrrolidine to a $5 \mathrm{wt} \%$ concentration. A 3:1 ratio of base (n-methyl pyrrolidone for QAPSf and 1-methyl imidazole for QIPSf) to chloromethyl groups was added to the solution. The solution was reacted at $60^{\circ} \mathrm{C}$ for 16 hours. The solution 
was then cast onto a glass plate and heated to $60^{\circ} \mathrm{C}$ for 12 hours to evaporate the solvent. Afterward the membranes were washed in DI water to remove any residual base.

Permeability measurements of organic acid anions through AEMs

Permeability of lactate was measured in an H-cell apparatus with each chamber having $250 \mathrm{~mL}$ volume and $12.57 \mathrm{~cm}^{2}$ cross-sectional active area. A $0.1 \mathrm{M}$ sodium lactate $/ 0.5 \mathrm{M}$ potassium nitrate (1:5 ratio) was loaded into the retentate chamber and a $0.5 \mathrm{M}$ potassium nitrate in the permeate chamber. The 1:5x ratio of potassium nitrate was intended to minimize osmotic pressure differences between the chambers. It is important to note that no change in water volume was observed during the experiment. Lactic acid concentration in the permeate were monitored at various time points over a 120-minute duration by HPLC. Permeability was calculated from Equation 1, where $V_{B}$ is the volume of the permeate chamber, $d$ is the membrane thickness, $\mathrm{C}_{\mathrm{A}}$ is the concentration of the retentate chamber, $\mathrm{A}$ is the active area of membrane, and $\frac{d C_{B}}{d t}$ is the acid crossover rate.

$$
P=\frac{V_{B} \times d}{C_{A} \times A} \times \frac{d C_{B}}{d t}
$$

\section{Ionic conductivity}

A 4-point conductivity cell consisting of four platinum wires housed in a polytetrafluoroethylene (PTFE) housing was used to measure in-plane AEM conductivity ( $\kappa)$. The probe was immersed in a supporting electrolyte that consisted of $0.1 \mathrm{~g} / \mathrm{L}$ lactic acid titrated with sodium hydroxide solution to a neutral $\mathrm{pH}$ of about 7 . The membrane resistance was determined using electrochemical impedance spectroscopy (EIS). A $1 \mathrm{~mA}$ perturbation in the frequency range of $100 \mathrm{kHz}$ to $1 \mathrm{~Hz}$ was used during galvanostatic EIS. The high frequency 
resistance extracted from the Nyquist plot was used to calculate the conductivity with Equation 3.

$$
\kappa=\frac{L}{R \cdot d \cdot W}
$$

$\kappa$ denotes the ionic conductivity, $\mathrm{L}$ denotes the distance between electrodes, $\mathrm{R}$ denotes the resistance of the membrane, $\mathrm{d}$ is the membrane thickness, and $\mathrm{W}$ is the width of the membrane.

Organic acid anion uptake of AEMs and calculating the equilibrium partitioning coefficient

The uptake of organic acids was measured using a gravimetric and quantitative NMR approach. This data was used to calculate the equilibrium partitioning coefficient $(K)$. The organic acid uptake experiment commenced with preparing a desired concentration of the organic acid ( $1 \mathrm{M}, 0.5 \mathrm{M}$, and $0.1 \mathrm{M}$ lactic acid) in $10 \mathrm{~mL}$ of deuterium. Then, $20 \mathrm{mg}$ of membrane (approx. $1.5 \mathrm{~cm} \mathrm{x} 4.0 \mathrm{~cm}$ ) was immersed in the prepared solution for 48 hours for equilibrium to occur. The membrane sample was removed from the solution, blot dried, and the dimensions and final mass were quickly measured. The absorbed acid content from gravimetric measurements was determined by Equation 4, where $\mathrm{C}_{\text {membrane }}$ is the mass of acid absorbed per mass of membrane, mequil is the mass of membrane after equilibrium uptake, and $m_{\text {dry }}$ is the original mass of membrane.

The membrane was then dissolved in $1 \mathrm{~mL}$ of a $9.6 \mathrm{mM}$ DSS/DMSO-d6 solution and ${ }^{1} \mathrm{H}$ NMR spectrums of each sample were acquired on a $400 \mathrm{MHz}$ Bruker spectrometer. Quantitative NMR (qNMR) was applied to calculate the lactate content in the dissolved membrane according to Equation 5, where m, I, N, M, P represent the mass, integrated area, number of hydrogen atoms, molecular mass, and purity of the acid and reference DSS internal standard, respectively. The reference peak at $\sim 0 \mathrm{ppm}$ was used to calibrate the DSS internal standard which corresponds 
to 3 methyl groups (i.e., $\mathrm{N}_{\text {ref }}=9$ ). The NMR peak corresponding to the methyl group of lactic acid $(\sim 1.2 \mathrm{ppm})$ was used to quantify lactic acid concentration (i.e., $\left.\mathrm{N}_{\mathrm{a}}=3\right)$. The equilibrium partitioning parameter, $\mathrm{K}$, between the organic acid and the membrane was then calculated using

\section{Equation 6.}

$$
\begin{gathered}
C_{\text {membrane }}=\frac{m_{\text {equil }}-m_{\text {dry }}}{m_{\text {equil }}}=\frac{m_{a}}{m_{\text {equil }}} \\
m_{a}=\frac{I_{a}}{I_{\text {ref }}} \times \frac{N_{\text {ref }}}{N_{a}} \times \frac{M_{a}}{M_{\text {ref }}} \times \frac{P_{\text {ref }}}{P_{a}} \times m_{\text {ref }} \\
K=\frac{C_{\text {membrane }}}{C_{\text {solution }}}
\end{gathered}
$$

\section{Ionic flux and permselectivity measurements using ED}

ED experiments were conducted using a custom-built stack consisting of a single cell pair and stainless-steel cathode and dimensionally-stable anode (DSA). Ion-exchange membranes (14 $\mathrm{mm}^{2}$ active area) were arranged to form an alternating pattern of anion and cation exchange membranes to form feed/diluate compartments of $\sim 2.5 \mathrm{~mm}$ thickness (see Figure 1a). Bipolar membranes were arranged to isolate interior chambers from the electrode rinse compartments. Experiments were conducted in continuous mode using a synthetic fermentation broth of $33 \mathrm{~g} \mathrm{~kg}^{-}$

${ }^{1}$ lactic acid, $1.25 \mathrm{~g} \mathrm{~kg}^{-1}$ sodium sulfate and $0.72 \mathrm{~g} \mathrm{~kg}^{-1}$ sodium phosphate for the diluate compartment and $10 \mathrm{~g} \mathrm{~kg}^{-1}$ sodium chloride for the concentrate compartment. The flow rate for each solution through the stack were $20 \mathrm{~mL} \mathrm{~min}^{-1}$ and the stack was operated at constant voltage with $1 \mathrm{~V}$ cell pair ${ }^{-1}$. Acid effluent concentrations were monitored by HPLC and the inorganic anions by ion-exchange chromatography (IC).

The ionic flux for lactic acid ( $\left.\mathrm{J}_{\text {lactic acid }}\right)$ and the contaminant molecules $\left(\mathrm{J}_{\text {contam; }}\right.$ i.e., competing anions of sulfate and phosphate) were determined from Equation 7. $\mathrm{J}_{\mathrm{i}}$ represents the 
flux of species $i$ captured in the concentrate chamber, $\mathrm{m}_{\text {conc }}$ is the total mass of species in the concentrate chamber, and $\frac{d C_{i}}{d t}$ is the change in concentration of species $i$ in the concentrate chamber. The transport numbers from Equation 8 were used to calculate the permselectivity of each species according to Equation 9. In these equations, $\mathrm{Clactic}_{\text {acid }}$ is the lactic acid concentration in the diluate chamber and $\mathrm{C}_{\text {contam }}$ is the contaminant species concentration in the diluate chamber. Energy consumption was calculated using Equation 10, where V is the constant voltage applied to the stack, I is the current for the stack, and $m$ denotes the mass of acid captured.

$$
\begin{gathered}
J_{i}=\frac{m_{\text {conc }}}{A} \frac{d C_{i}}{d t} \\
t_{i}=\frac{J_{i}}{\sum J_{\text {total }}} \\
P_{\text {contaminate }}^{\text {Lactic Acid }}= \\
=\frac{t_{\text {Lactic Acid }} / t_{\text {contam }}}{C_{\text {Lactic Acid }} / C_{\text {contam }}}=\frac{J_{\text {Lactic Acid }} \cdot C_{\text {contam }}}{J_{\text {contam }} \cdot C_{\text {Lactic Acid }}} \\
E=\frac{V \int I d t}{m}
\end{gathered}
$$

\section{HPLC and ion-chromatography analysis}

Lactic acid concentration was measured by high performance liquid chromatography (HPLC, 717 Plus Autosampler; Waters, Milford, MA) equipped with a refractive index detector. Analysis was performed with $5 \mathrm{mM}$ sulfuric acid mobile phase and $0.6 \mathrm{~mL} \mathrm{~min}{ }^{-1}$ flow rate and $10 \mu \mathrm{L}$ injection volume. Sulfate and phosphate anion concentrations were measured with ionexchange chromatography (IC, 882 Compact IC plus; Metrohm, Riverview, FL) equipped with chemical and $\mathrm{CO}_{2}$ suppression systems. Analyses were performed with Metrosep A Supp 5 150/4.0 analytical and guard columns, $3.2 \mathrm{mM} \mathrm{Na}_{2} \mathrm{CO}_{3} / 1.0 \mathrm{mM} \mathrm{NaHCO}_{3}$ as the eluent, a flow rate of $0.7 \mathrm{~mL} \mathrm{~min}^{-1}$, and $20 \mu \mathrm{L}$ sample loop and injection volumes. 
Nuclear overhauser effect spectroscopy (NOESY) NMR

The ${ }^{1} \mathrm{H} 2 \mathrm{D}$ NOESY data was acquired on a $400 \mathrm{MHz}$ Bruker spectrometer at $300 \mathrm{~K}$ temperature using samples prepared with deuterated dimethyl sulfoxide (d6-DMSO) as the solvent. Highly concentrated sample ( $25 \mathrm{mg}$ polymer per $1 \mathrm{~mL}$ of d6-DMSO) were prepared to reduce the relative solvent and water peak heights. To improve the signal-noise ratio, the number of scans per slice was increased from 4 scans (default) to 64 scans. The total number of slices in the 2D spectrum was 256 slices. ${ }^{1} \mathrm{H}$ NMR and ${ }^{1} \mathrm{H}$ 2D NOESY NMR was also performed on sodium lactate $(0.5 \mathrm{M})$ in d6-DMSO to clearly identify the sodium lactate signals/peaks. The 2D NOESY NMR experiments were performed on the AEM samples in the chloride and lactate counterion form. Ion-exchange of the chloride counterions with the lactate counterions occurred by immersing the AEM samples in $0.5 \mathrm{M}$ sodium lactate in deionized water solution for 48 hours. The mixing time $\left(T_{m i x}\right)$ for the NOESY scans was selected to be sufficiently long $(500 \mathrm{~ms})^{49}$ to enable the observation of any through-space NOE correlations.

\section{Computation molecular dynamics methods}

Both classical molecular dynamics simulations using LAMMPS ${ }^{50}$ program and electronic structure calculations using Gaussian 09 suite of programs ${ }^{51}$ were carried out on model systems to investigate the affinity of lactate anions towards benzyl imidazolium and quaternary benzyl ammonium groups on PSf backbones. For MD simulations, the PSf with tethered imidazolium or quaternary ammonium groups consisted of five repeating units. A lactate anion was introduced to each tethered cation moiety as a counterion. Interactions of lactate ions with each PSf AEM were simulated in a cubic box consisting 40 cation/anion pairs solvated in 800 water molecules using a conventional non-reactive force-field OPLSAA ${ }^{52}$. A detailed description of the MD simulation 
setup is provided in the SI. Binding energy of a lactate anion with an imidazolium cation and an ammonium cation were determined by carrying out electronic structure calculations on representative clusters in the gas phase at the B3LYP/6-31+g(d) level of theory. For these cluster calculations the structures shown in Figure S8a and S8b were used to represent the cation groups chemical structures in QIPSf and QAPSf polymers respectively. The xyz coordinates of the geometry optimized input structures (Figures S8a and S8b) and the optimized IM-lactate/AMlactate structures (Figures 5a and $\mathbf{5 b}$ ) are provided in Tables $\mathbf{S 1}$ and $\mathbf{S 2}$ in the $S I$.

\section{DATA AVAILABILITY}

Data reported in the manuscript are provided in the Penn State Data Commons repository. 


\section{REFERENCES}

1. Lister, T. E. et al. Low-Temperature Electrochemical Upgrading of Bio-oils Using Polymer Electrolyte Membranes. Energy and Fuels 32, 5944-5950 (2018).

2. Sauer, M., Mattanovich, D. \& Marx, H. Microbial production of organic acids for use in food. in Microbial Production of Food Ingredients, Enzymes and Nutraceuticals 288-320 (Elsevier Ltd., 2013). doi:10.1533/9780857093547.2.288.

3. Survase, S. A., Bajaj, I. B. \& Singhal, R. S. Biotechnological production of vitamins. Food Technology and Biotechnology vol. 44 381-396 https://www.ftb.com.hr/index.php/archives/76-volume-44-issue-no-3/388 (2006).

4. Handojo, L. et al. Electro-membrane processes for organic acid recovery. $R S C A d v$. 9 , 7854-7869 (2019).

5. Komesu, A., Oliveira, J. A. R. de, Martins, L. H. da S., Wolf Maciel, M. R. \& Maciel Filho, R. Lactic Acid Production to Purification: A Review. BioResources 12, (2017).

6. Othman, M., Ariff, A. B., Rios-Solis, L. \& Halim, M. Extractive Fermentation of Lactic Acid in Lactic Acid Bacteria Cultivation: A Review. Front. Microbiol. 8, 2285 (2017).

7. Pal, P., Sikder, J., Roy, S. \& Giorno, L. Process intensification in lactic acid production: A review of membrane based processes. Chem. Eng. Process. Process Intensif. 48, 15491559 (2009).

8. Huang, C., Xu, T., Zhang, Y., Xue, Y. \& Chen, G. Application of electrodialysis to the production of organic acids: State-of-the-art and recent developments. Journal of Membrane Science vol. 288 1-12 (2007).

9. Da Silva, A. H. \& Miranda, E. A. Adsorption/desorption of organic acids onto different adsorbents for their recovery from fermentation broths. J. Chem. Eng. Data 58, 14541463 (2013).

10. National Academies of Sciences, E. and M. A Research Agenda for Transforming Separation Science. A Res. Agenda Transform. Sep. Sci. (2019) doi:10.17226/25421.

11. Kim, Y. H. \& Moon, S. H. Lactic acid recovery from fermentation broth using one-stage electrodialysis. J. Chem. Technol. Biotechnol. 76, 169-178 (2001).

12. Madzingaidzo, L., Danner, H. \& Braun, R. Process development and optimisation of lactic acid purification using electrodialysis. J. Biotechnol. 96, 223-239 (2002).

13. Li, Y. \& Shahbazi, A. Lactic Acid Recovery From Cheese Whey Fermentation Broth Using Combined Ultrafiltration and Nanofiltration Membranes. in Twenty-Seventh Symposium on Biotechnology for Fuels and Chemicals 985-996 (Humana Press, 2007). doi:10.1007/978-1-59745-268-7 83.

14. Sikder, J., Roy, M., Dey, P. \& Pal, P. Techno-economic analysis of a membraneintegrated bioreactor system for production of lactic acid from sugarcane juice. Biochem. Eng. J. 63, 81-87 (2012).

15. Lin, Y. J., Hestekin, J. A., Henry, M. P. \& Sather, N. Chapter 9:Bioprocessing of Costcompetitive Biobased Organic Acids. RSC Green Chem. 2016-January, 190-214 (2015).

16. Zheleznov, A., Windmöller, D., Körner, S. \& Böddeker, K. W. Dialytic transport of carboxylic acids through an anion exchange membrane. J. Memb. Sci. 139, 137-143 (1998).

17. Pourcelly, G., Tugas, I. \& Gavach, C. Electrotransport of sulphuric acid in special anion exchange membranes for the recovery of acids. J. Memb. Sci. 97, 99-107 (1994).

18. Lorrain, Y., Pourcelly, G. \& Gavach, C. Transport mechanism of sulfuric acid through an 
anion exchange membrane. Desalination 109, 231-239 (1997).

19. Nikonenko, V., Lebedev, K., Manzanares, J. A. \& Pourcelly, G. Modelling the transport of carbonic acid anions through anion-exchange membranes. Electrochim. Acta 48, 36393650 (2003).

20. Koter, S. \& Kultys, M. Electric transport of sulfuric acid through anion-exchange membranes in aqueous solutions. J. Memb. Sci. 318, 467-476 (2008).

21. Le, X. T. Contribution to the study of properties of Selemion AMV anion exchange membranes in acidic media. Electrochim. Acta 108, 232-240 (2013).

22. Pismenskaya, N., Nikonenko, V., Volodina, E. \& Pourcelly, G. Electrotransport of weakacid anions through anion-exchange membranes. Desalination 147, 345-350 (2002).

23. Gally, C., García-Gabaldón, M., Ortega, E. M., Bernardes, A. M. \& Pérez-Herranz, V. Chronopotentiometric study of the transport of phosphoric acid anions through an anionexchange membrane under different pH values. Sep. Purif. Technol. 238, 116421 (2020).

24. Akgemci, E. G., Ersöz, M. \& Atalay, T. Transport of Formic Acid Through Anion Exchange Membranes by Diffusion Dialysis and Electro-Electro Dialysis. http://dx.doi.org/10.1081/SS-120027407 39, 165-184 (2010).

25. Kim, D. H. et al. Development of thin anion-exchange pore-filled membranes for high diffusion dialysis performance. J. Memb. Sci. 447, 80-86 (2013).

26. Chandra, A., Bhuvanesh, E., Mandal, P. \& Chattopadhyay, S. Surface modification of anion exchange membrane using layer-by-layer polyelectrolytes deposition facilitating monovalent organic acid transport. Colloids Surfaces A Physicochem. Eng. Asp. 558, 579590 (2018).

27. Ji, W. et al. Self-organized nanostructured anion exchange membranes for acid recovery. Chem. Eng. J. 382, 122838 (2020).

28. $\mathrm{Wu}, \mathrm{Y}$. et al. QPPO/PVA anion exchange hybrid membranes from double crosslinking agents for acid recovery. J. Memb. Sci. 428, 95-103 (2013).

29. Kutz, R. B. et al. Sustainion Imidazolium-Functionalized Polymers for Carbon Dioxide Electrolysis. Energy Technol. 5, 929-936 (2017).

30. Yang, H., Kaczur, J. J., Sajjad, S. D. \& Masel, R. I. Electrochemical conversion of CO2 to formic acid utilizing Sustainion ${ }^{\mathrm{TM}}$ membranes. J. CO2 Util. 20, 208-217 (2017).

31. Liu, Z., Yang, H., Kutz, R. \& Masel, R. I. CO 2 Electrolysis to CO and O 2 at High Selectivity, Stability and Efficiency Using Sustainion Membranes . J. Electrochem. Soc. 165, J3371-J3377 (2018).

32. Hagesteijn, K. F. L., Jiang, S. \& Ladewig, B. P. A review of the synthesis and characterization of anion exchange membranes. J. Mater. Sci. 2018 5316 53, 1113111150 (2018).

33. Arges, C. G. \& Zhang, L. Anion Exchange Membranes' Evolution toward High Hydroxide Ion Conductivity and Alkaline Resiliency. ACS Appl. Energy Mater. 1, 29913012 (2018).

34. Su, X. et al. Asymmetric Faradaic systems for selective electrochemical separations. Energy Environ. Sci. 10, 1272-1283 (2017).

35. Su, X. et al. Electrochemically-mediated selective capture of heavy metal chromium and arsenic oxyanions from water. Nat. Commun. 201891 9, 1-9 (2018).

36. Kim, K. et al. Electrochemical approaches for selective recovery of critical elements in hydrometallurgical processes of complex feedstocks. iScience 24, 102374 (2021).

37. White, J. F., Holladay, J. E., Zacher, A. A., Frye, J. G. \& Werpy, T. A. Challenges in 
Catalytic Manufacture of Renewable Pyrrolidinones from Fermentation Derived Succinate. Top. Catal. 20145717 57, 1325-1334 (2014).

38. Cadena, C. et al. Why is $\mathrm{CO} 2$ so Soluble in Imidazolium-Based Ionic Liquids? J. Am. Chem. Soc. 126, 5300-5308 (2004).

39. Carlisle, T. K., Bara, J. E., Lafrate, A. L., Gin, D. L. \& Noble, R. D. Main-chain imidazolium polymer membranes for $\mathrm{CO} 2$ separations: An initial study of a new ionic liquid-inspired platform. J. Memb. Sci. 359, 37-43 (2010).

40. Bara, J. E. et al. Guide to CO 2 separations in imidazolium-based room-temperature ionic liquids. Ind. Eng. Chem. Res. 48, 2739-2751 (2009).

41. Robeson, L. M. Correlation of separation factor versus permeability for polymeric membranes. J. Memb. Sci. 62, 165-185 (1991).

42. Claridge, T. D. W. High-Resolution NMR Techniques in Organic Chemistry: Third Edition. High-Resolution NMR Tech. Org. Chem. Third Ed. 1-541 (2016) doi:10.1016/C2015-0-04654-8.

43. Arges, C. G. \& Ramani, V. Two-dimensional NMR spectroscopy reveals cation-triggered backbone degradation in polysulfone-based anion exchange membranes. Proc. Natl. Acad. Sci. U. S. A. 110, 2490-2495 (2013).

44. Arges, C. G. \& Ramani, V. Investigation of Cation Degradation in Anion Exchange Membranes Using Multi-Dimensional NMR Spectroscopy. J. Electrochem. Soc. 160, F1006-F1021 (2013).

45. Kamcev, J., Paul, D. R., Manning, G. S. \& Freeman, B. D. Ion Diffusion Coefficients in Ion Exchange Membranes: Significance of Counterion Condensation. Macromolecules 51, 5519-5529 (2018).

46. Kamcev, J., Paul, D. R. \& Freeman, B. D. Ion Activity Coefficients in Ion Exchange Polymers: Applicability of Manning's Counterion Condensation Theory. Macromolecules 48, 8011-8024 (2015).

47. Lei, Q. et al. Counterion condensation or lack of solvation? Understanding the activity of ions in thin film block copolymer electrolytes. J. Mater. Chem. A 8, 15962-15975 (2020).

48. Arges, C. G., Parrondo, J., Johnson, G., Nadhan, A. \& Ramani, V. Assessing the influence of different cation chemistries on ionic conductivity and alkaline stability of anion exchange membranes. J. Mater. Chem. 22, 3733-3744 (2012).

49. Jenkins, J. E., Hibbs, M. R. \& Alam, T. M. Identification of Multiple Diffusion Rates in Mixed Solvent Anion Exchange Membranes Using High Resolution MAS NMR. ACS Macro Lett. 1, 910-914 (2012).

50. Plimpton, S. Fast Parallel Algorithms for Short-Range Molecular Dynamics. J. Comput. Phys. 117, 1-19 (1995).

51. Frisch, M. et al. Gaussian 09 (Revision A02). Gaussian Inc. Wallingford CT (2009).

52. William L. Jorgensen, *, David S. Maxwell, and \& Tirado-Rives, J. Development and Testing of the OPLS All-Atom Force Field on Conformational Energetics and Properties of Organic Liquids. J. Am. Chem. Soc. 118, 11225-11236 (1996). 


\section{ACKNOWLEDGEMENTS}

The submitted manuscript has been created jointly by UChicago Argonne, LLC, Operator of Argonne National Laboratory (“Argonne”). Argonne, a US Department of Energy Office of Science laboratory, is operated under contract no. DE-AC02-06CH11357. M.L Jordan's and Y.J. Lin's effort was financially sponsored by the U.S. Department of Energy's (DOE's) Bioenergy Technologies Office (BETO). C.G. Arges, T. Kulkarni, D.I. Senadheera, and R. Kumar's work was supported by the U.S. Department of Energy, Office of Science, Office of Basic Energy Sciences Separation Science program under Award No. DE-SC0018989. M.L. Jordan acknowledges support from the U.S. Department of Energy Office of Science Graduate Student Research (SCGSR) Program, National Science Foundation Graduate Research Fellowship and from the Jack Kent Cooke Foundation. This work used Penn State's Department of Chemistry NMR spectrometers.

\section{AUTHOR CONTRIBUTIONS}

M.L.J., C.A., and Y.L. conceived the project. M.L.J. performed polymer synthesis, membrane characterization and device-level studies. T.K. carried out 2D NOESY experiments. D.I.S. and R.K. conducted MD and DFT simulations. M.L.J., C.A., D.I.S., and R.K. drafted and edited the manuscript.

\section{COMPETING INTERESTS}

There are no conflicts of interest to declare. 


\section{MATERIALS AND CORRESPONDENCE}

Correspondence and material requests can be directed toward C.A. or Y.L. 


\section{Supplementary Files}

This is a list of supplementary files associated with this preprint. Click to download.

- SIlmidazoliumtypeanionexchangemembranesforimprovedorganicacidtransportsubmitted.pdf 